\title{
Modeling GMPLS and Optical MPLS Networks
}

\section{Christiansen, Henrik Lehrmann; Wessing, Henrik}

Published in:

10th International Conference on Telecommunications, 2003. ICT 2003.

Link to article, DOI:

10.1109/ICTEL.2003.1191237

Publication date:

2003

Document Version

Publisher's PDF, also known as Version of record

Link back to DTU Orbit

Citation (APA):

Christiansen, H. L., \& Wessing, H. (2003). Modeling GMPLS and Optical MPLS Networks. In 10th International Conference on Telecommunications, 2003. ICT 2003. IEEE. https://doi.org/10.1109/ICTEL.2003.1191237

\section{General rights}

Copyright and moral rights for the publications made accessible in the public portal are retained by the authors and/or other copyright owners and it is a condition of accessing publications that users recognise and abide by the legal requirements associated with these rights.

- Users may download and print one copy of any publication from the public portal for the purpose of private study or research.

- You may not further distribute the material or use it for any profit-making activity or commercial gain

- You may freely distribute the URL identifying the publication in the public portal

If you believe that this document breaches copyright please contact us providing details, and we will remove access to the work immediately and investigate your claim. 


\title{
Modeling GMPLS and optical MPLS networks
}

\author{
Henrik Christiansen and Henrik Wessing \\ COM \\ Bldg. 345V, Technical University of Dent lark \\ $2800 \mathrm{Kgs}$. Lyngby \\ Denmark \\ E-mail: hc@com.dtu.dk
}

\begin{abstract}
A consequence of migrating the existing Internet architecture to an all-optical one is that the network will consist of a mixture of equipment, ranging from electrical routers to all-optical packet switches. Hence, future networks will consist of multiple domains employing different technologies: The MPLS concept is : attractive because it can work as a unifying control structure : covering all technologies. This paper describes how a novel . scheme for optical MPLS and circuit switched GMPLS based networks can incorporated in such multi-domain, MPLS-based scenarios and how it could be modeled. Network-nodes supporting GMPLS the proposed novel scheme is implemented and . routing and path setup is demonstrated.

\section{Introduction}

In the old days, the vision was to create one single technology for multi service networks. This was one of the drivers behind developing and deploying ATM. However, the technologies being developed today are of a different nature. It is no Ionger likely with a network based on one single technology, simply because the vast amount of equipment in e.g., the global Intemet makes instant upgrade/replacement impossible. Migration to future technologies will be seen as islands popping up and this gradual upgrade creates heterogeneous networks consisting of a number of different technologies. Currently, for instance, optical technologies are being introduced into the networks, but electrical routers/switches are still present. Thus, the networks of the future will be multi technology, multi service networks as sketched in Figure 1. Add to that the requirements of traffic engineering capabilities and you will end up with a very complex network.
\end{abstract}

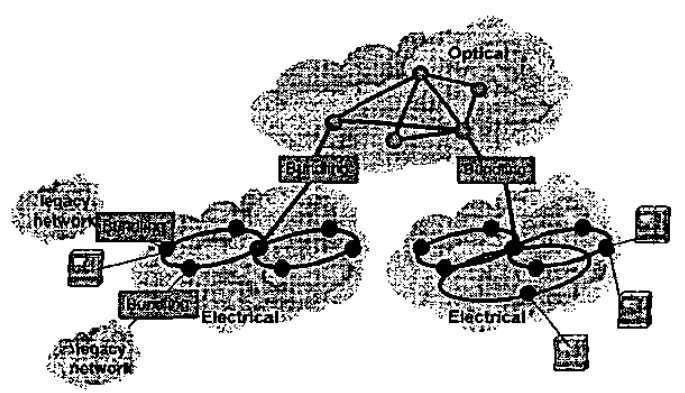

Figure I: A multi-domain network comprising different technologies

This has had an impact on the structure of modern networks, but also this has created a requirement for special adaptation devices that are able to propagate traffic between network domains running different technologies and for a common control plane structure able to inify all these technologies and create a useful network. A close $r$ look at the adaptation devices can be found in [Chr2001]. In th: s paper the emphasis is on the control part of the network.

This paper is org anized as follows. Firstly, a brief MPLS tutorial is provided. The I the limitations for introducing optical MPLS are reviewed, an 1 two possible approaches are presented. One is a novel approact, which avoids heäder modification, and the other is GMPLS, The integration of those technologies is treated and it is describe $J$ how to model these combined MPLS / ; GMPLS networl s. The GMPLS as well as the optical MPLS OPNET model a e then presented along with some simulation results that verif.' the functionality and illustrate how the models interoperates wit 1 the build in OPNET MPLS riodel.

\section{MPLS based concepts}

This section intri duces the MPLS networking concept suitable for electrical pac zet switching. The use of the MPLS concept with all-optical network nodes is considered arid a novel scheme and the GMPLS' 'oncept is presented as solutions to the faced problems.

\section{Basic properies of MPLS}

MPLS [Ros2001 1 ] is a networking concept that is based mainly on a shift of all c smplex functionality to the edge of the network, leaving only sim ,le operation for the core network and hence enabling fast and efficient operation. The control plane (that takes care of e.g. routing) and switching (packet forwarding) are completely decol pled, which yields the advantageous property that they can be c hosen independently. This is the main reason why we in this pi per can consider routing and structural issues without treating $i$.g., packet forwarding explicitly. MPLS is designed as a pure "zverything over everything' concept, hence its name. In reality, owever, its predominant use and the majority of standardizatio i work are focused on carrying IP traffic with MPLS, which is ilue to the importance of the ubiquitous Internet. In MPLS packets are forwarded along routes called Label Switched Paths ( $S P s)$ that may be determined by routing protocols based on pre Jefined traffic classes called Forward Equivalent Classes (FEC 's). An FEC can be equivalent to a single entry in a conventional IP routing table or it can be an aggregation of multiple entries. An FEC can also be specified based on a number of additional onstraints such as originating address, receiving port number ind QoS parameters. These LSPs are defined in the switches by u.sing labels, which are distributed by a Label Distribution Proticol (LDP) responsible for mapping between routing and switc aing. The MPLS standard doesn't specify one specific label disı ribution protocol; it just highlights the required properties. Curre: tlly, four protocols of which two are new and two are modifica'ions of existing protocols are mentioned in the standards [And2( 01][Rekh2000][Jamo1 999][Brad1997]. 
One of the major benefits of the MPLS concept is its ability to perform traffic engineering, i.e., to be able to control how traffic tlows through the network, which is one of the prerequisites for providing QoS guarantees on connections. Generally, traffic engineering implies to route along non-shortest paths and utilizes Constraint Based Routing (CBR) where the routes are calculated subject to performance- and administrative constraints, which are assigned by the network management system, based on e.g., traffic measurements.

In MPLS, switches are generally called Label Switch Routers (LSRs). Ingress edge LSRs take care of attaching short, fixed length labels to packets when they enter the MPLS domain, which includes the non-trivial task of determining to which FEC a given packet belongs. Within the core of the network forwarding will be based on the label only, and before leaving the MPLS domain packets have their label removed by the egress edge LSR, as it is sketched in Figure 2.

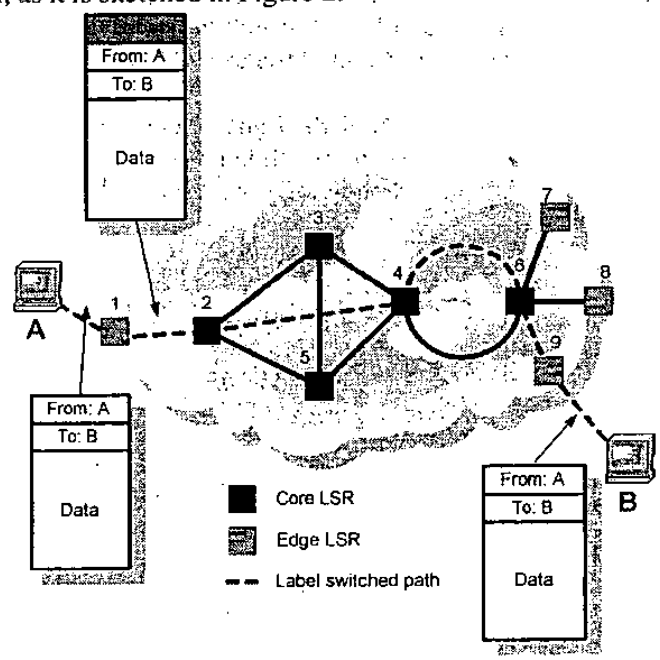

Figure 2: The label is used only within one MPLS domain. By attaching different labels at the ingress $L S R$, different routes through the network for the same destination can be selected, which allows for traffic engineering.

The labels are generally not kept constant along an LSP and thus a path through the network is defined by a sequence of labels, all of which are assigned by the LDP. Within the core switches only the labels are examined, and what distinguishes this method from that of conventional IP routing are the loose coupling between the label and the destination address as well as the lookup scheme within the switches themselves. The labels used by MPLS require exact match in the lookup tables, which is a much simpler operation than LPM [Rekh1995]. I.e., OSPF would build a routing table is each LSR and based on this information and possibly additional information the label distribution protocol builds another table in which the label is used as the key. The outcome of a table lookup is information about outgoing port number and the outgoing label, which is used to replace the label contained within the packet as well as expediting the packet to the designated output port. The label replacement operation is usually called label swapping and is the most common packet modification operation in MPLS. In addition, when working with multiple domains in a network, the single label might be replaced by a stack of labels with only the top label being used within one particular domain. At domain boundaries label swapping is insufficient and must be exchanged for more complex operations such as label pushing and popping.

\section{Optical MPLS}

MPLS was designed for packet switched networks. However, when considering all-optical devices, packet switching using header modification is not yet a mature technology. Even though switching of optical signals potentially is done with very high bit rates [Dan 1997, Hun2000, Chi1998], the approach is facing several problems. Regeneration of the signal through $2 \mathrm{R}$ or $3 \mathrm{R}$ regeneration is required if several switches are cascaded

[Wol1999] and buffering of packets and optical label swapping are two challenges that are only solved in the labs, even though attempts have been done to reduce the buffer requirements by utilizing the wavelength dimension [Dan 1997]:

\section{Key identification}

As previously described, header modification is a main technological limitation for introducing optical MPLS network. This problem is addressed in the key identification scheme [Wess001][Chr2002], where the requirements to the optical layer are reduced. The concept of the scheme is shown in Figure 3 , for a nctwork comprising two edge nodes and three core nodes. Each node is initially assigned a unique so-called key.

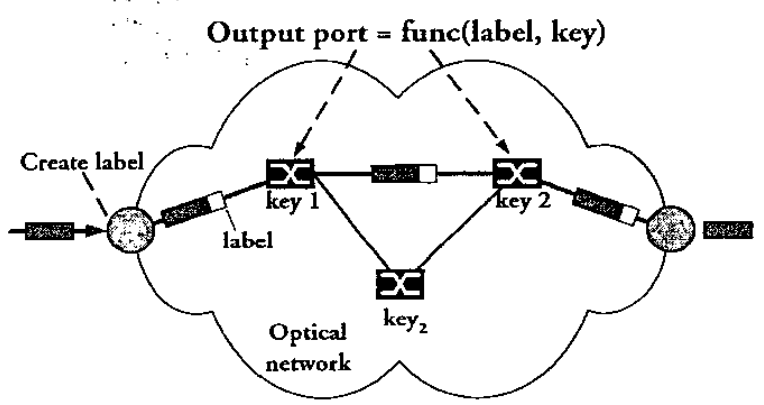

Figure 3: Concept of the key identification approach. The label, created at the edge node, is used together with a mathematical function to identify the output port in each core MPLS node.

It is desired to route the packet through the core nodes represented with key 1 and key 2 . This is achieved by creating a label at the ingress node, and by using this label and the node specific keys each core node calculates the outgoing port by a function on the label and the key.

The mathematical function is based on the Chinese Remainder Theorem [Cormen], which states that - with some restrictions it is possible to define two independent arrays of integers of same length and combine those to a single scalar, which we will use as the label.

Then, by a simple modulo function on the label (the scalar) and an integer from the first array, the result is the value from the other array. Hence, by defining the first array as the keys for the nodes along the path and the second array as the desired output ports for the nodes, then the label is created and at each node the correct output port is simply calculated. The only restriction is that all the keys should be pair-wise relative primes. 
As the same label is used at all the nodes, it is not necessary to modify the header along the path. Hence, optical header modification is avoided as the label is only created and removed at the edge LSRs. The scheme differs from "normal" MPLS as the full switching information is carried within the header. This might reduce the scalability of the scheme for very large network sizes, but on the other hand the use header modification and maintenance of an LDP is avoided.

\section{GMPLS}

GMPLS is a generalization of MPLS that allows a seamless integration of a multitude of technologies, especially circuit switched systems, with packet switched networks. Thus, interfacing with traditional telecom TDM systems (e.g. SONET /

$\therefore$ SDH) and wavelength routed optical networks is possible with the use of GMPLS. GMPLS is in widespread use and have been implemented by several manufacturers [Ber2002].

:
In contrast to optical packet switching technologies, the technologies for optical circuit switching are far more accessible in the core network. By using mixed-technology, multi domain networks the advantages of different technologies can be combined. The problem is normally that a unified control and man-s agement structure is lacking. However, by integrating MPLS, key-MPLS and GMPLS a number of advantages are significant... The integration is depicted in Figure 4 where the big cloud de: notes the.MPLS based domain and the smaller clouds are islands : of key-MPLS and GMPLS sub-domains.

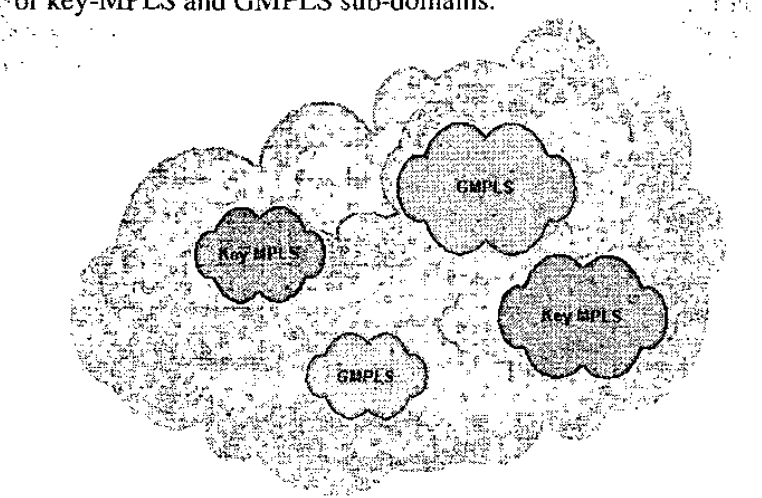

Figure 4: GMPLS in a typical usage scenario where GMPLS is used as 'islands' in the network.

A unified control and management structure can be uscd for the fult cloud. This enables support of traffic engineering even though different underlying physical layers are used.

Furthermore the advantages for both circuit- and packet switched networks is combined, which is advantageous as it offers:

- Traffic engineering capabilities,

- High capacity core

- Flexible, controllable edge

- Protocol independence (i.e., e.g. IP interoperability)

Hence GMPLS for circuit switched networks while allowing a management structure similar to standard MPLS.

\section{Modeling and integration}

The models in th is paper have been made with OPNET modeler 8.0 and the MPL 5 model suite. The MPLS model has been extended and modi ied in order to create GMPLS and key-ID network elements.

\section{Modeling GNIPLS}

Real GMPLS ne works are highly complex and may cover devices such as opl ical wavelength switches and SONET network nodes, i.e. GMPJ $S$ can operate with as well electronic as optical technologies. He rce, GMPLS networks can get very complex since a multitude of technologies are hidden there, implying a vast number of $\mathrm{p}$ :otocols, devices and configuration options. The real-life net / ork must be simplified greatly in order to bc able to build a $m$ del that can produce results within an acceptable timeframe $\downarrow$ brute-force modeling methodology that just tries to model thi real network in every detail is inappropriate. Below the goals or the simulation arc identified and based on that the simplifie 1 simulation model can be set up. Obviously, the model must $t$ e simple enough to achieve the identified goals, while representir $g$ a fair model of the real network

\section{Requirements $t_{1}$, the model}

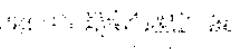

The goal of this imulation study is to build a model of how GMPLS interact! with an MPLS based network With the model it should be poss ble to measure/study:

- Call setilp probability

- Optical 'ignal quality

- Networl! topology / routing issues

- Label le igth (when model is used for key-MPLS) A list of input pa ameters is provided below:

\begin{tabular}{|l|l|}
\hline Attribute & Description \\
\hline Topology genera ion parameters \\
\hline - Number of nod ss & $\begin{array}{l}\text { Size and connectivity of the } \\
\text { network }\end{array}$ \\
\hline - Number of link & \\
\hline - Maximum dist nce & Bandwidth constraints \\
\hline Path constraints & SONET/ pure optical \\
\hline Type of network & \\
\hline
\end{tabular}

\section{OPNET implem sntation}

The MPLS mode has been extended/modified in order to create a GMPLS networ k element that can be built into MPLS network This GMPLS mo lels element represents the entire GMPLS network, i.e. a comp ete topology can be built with this single node. Figure 4 illustrates how the GMPLS network can interoperate with MPLS devic ss, i.e., LSPs can be setup through the GMPLS domain in this mi red environment. . 


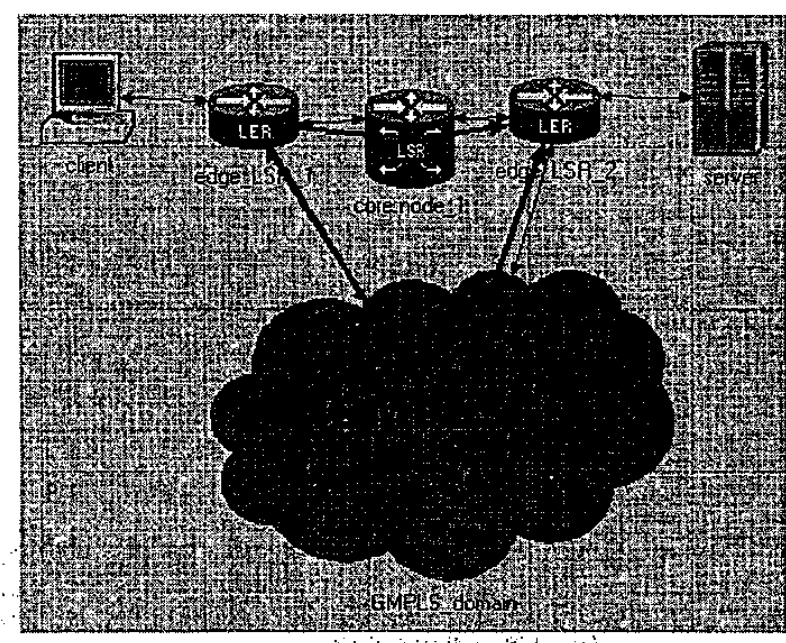

Figure 5: A GMPLS model, which can interoperate with MPLS, has been built into OPNET

More details of the implemented model.

In order to minimize the modifications needed in the OPNET code, GMPLS has been implemented as a separate process within the network nodes. The LDP process has then just been modified to detect whether this GMPLS process is present or not (and hence whether this is a MPLS or GMPLS node)

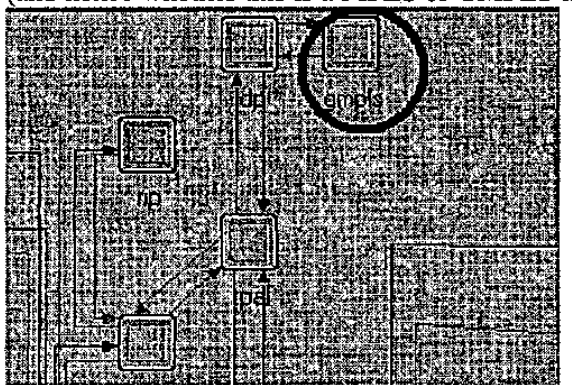

Figure 6: GMPLS has been implemented as a separate process in the MPLS node model

The details of the process model is shown below (figure 6)

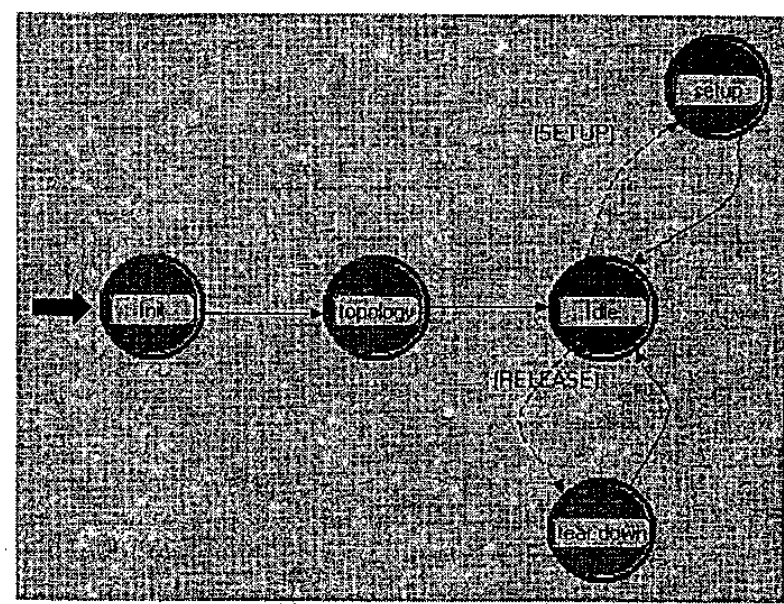

Figure 7: The GMPLS process model

$$
\text { ....... }
$$

Topology generation is performed by using the Route package in OPNET. The GMPLS implementations allows for either topology import from a file or generation of arbitrary topologies based on a specification-of the networks size (number of nodes and links). Modeling network topologies has been studied by a number of researchers [Zeg1996] [Fen2000] and it has been shown that the topologies have an impact on the network behavior. The topologies generated are suited to model an optical WDM network, i.e. the capacities of each link is given as a number of wavelengths. The actual capacity (i.e., bit rate) of each wavelength is not modeled explicitly. This is not necessary when path setup is considered as in this study.

The setup state tries to find a route through the network. One path requires one available wavelength from source to destination node. An attempt is made to find the shortest possible path though the network. This minimizes the overall capacity consumption of the oath and moreover (id the network is build from optical cross-connects) maximizes the signal quality. If the network possesses insufficient resources, the setup request is rejected.

Release request causes all resources associated with a given path to be released and they thus become available for future call setup requests.

Simulation results

This section contains results from simulations on the GMPLS model.

Now, let's try to arbitrarily generate network topologies. The results shown below are obtained for a network consisting of 20 nodes randomly (uniformly distributed) interconnected by 40 links. In total approximately 1750 setup requests were sent to this network. The paths are then active for a random time and then torn down.

Figure 8 shows the number of LSPs in the network. Paths setup is accomplished in the following way: The edge of the GMPLS domain receives the setup request from the surrounding MPLS network. Then an attempt is made to route the call though the GMPLS domain is made. To mimic all kinds of setup requests, 
two nodes in the GMPLS network are chosen at random and then an attempt to find a route to the destination is made.

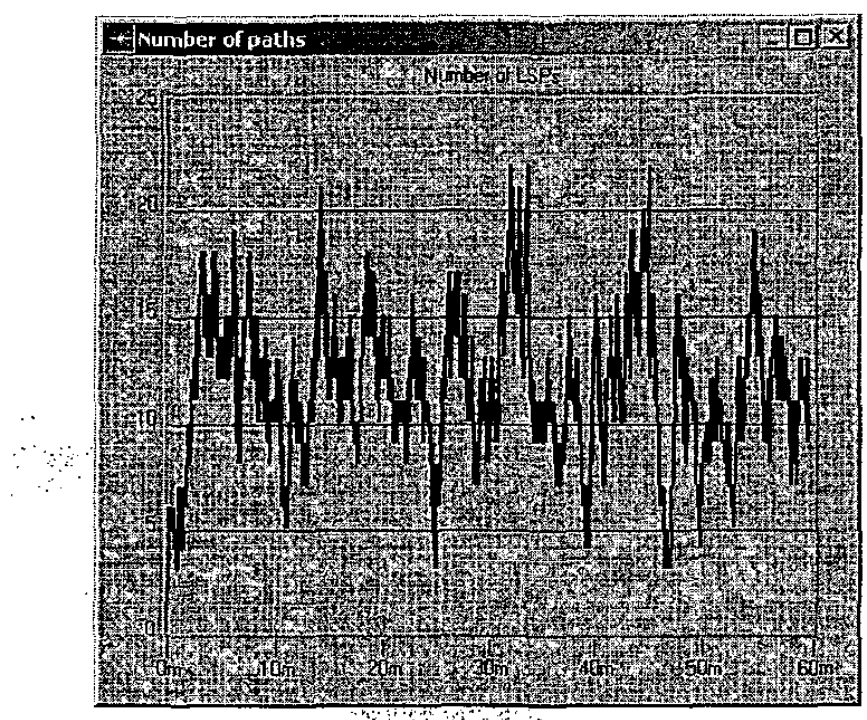

Figure 8: The number of established LSPSvaries during the simulation.

$$
\because \because \because \because
$$

In case no route exists the call is blocked, i.e., there is always a chance of a connection setup request being rejected. Figure 9 shows the rejection probability (rejected call / setup requests) for this network. Obviously the calculated probability gets more and more accurate with increasing number of calls. As can be seen, after 20 minutes, initial transients have gone. Hence to obtain a useful value for the call rejection probability at least 20 minutes should be simulated.

The path length varies depending on traffic load and network topology. The length (in number of hops) of the route impacts the OSNR of the signal. Hence for some OXC technologies, there can be an additional constraint (in addition to bandwidth requirements) on the path length. Figure 12 shows that for this particular network the path length varies from 2 to 7 hops.
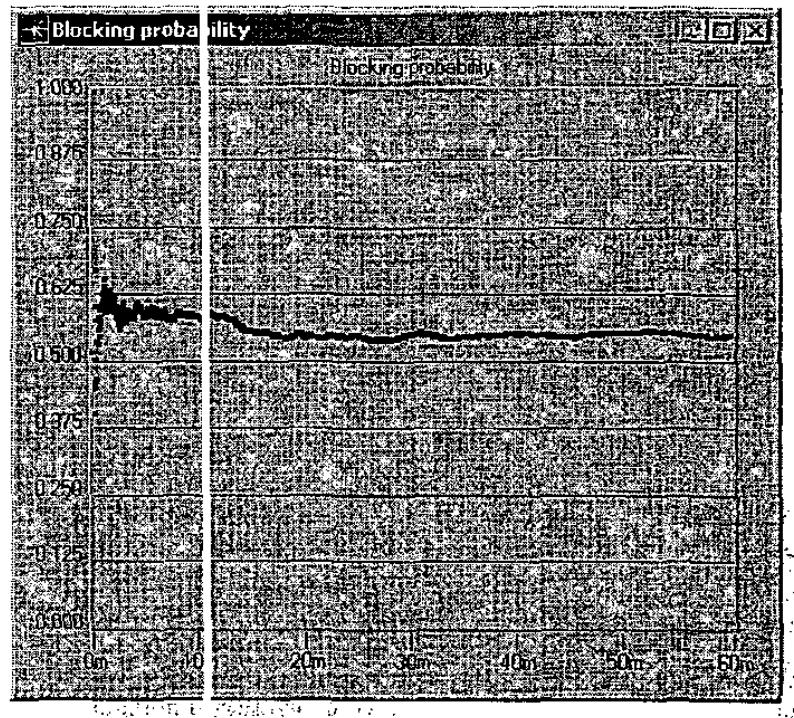

Figure 9: The reje tion (blocking) probability for a network consisting of 20 nodes and 4 links

If the size of the letwork is varied the results are as shown below (mean murnt er of pattis or LSPs, rejected calls and path length). In the si niulations, networks with between 10 and 30 nodes were gene ated. :All simulations are bases on approximately 500 call $\varsigma$ ətùps (per network size). Each graph is based on 55 simulation :

Figure 10 shows how the average number of simultaneous paths (LSPs) in the net work depends on the network size. As the number of calls is the same for all scenarios, these results are directly comparable to th': rejection probability shown below (figure 14). Clearly, lower re ection probability implies more LSPs.

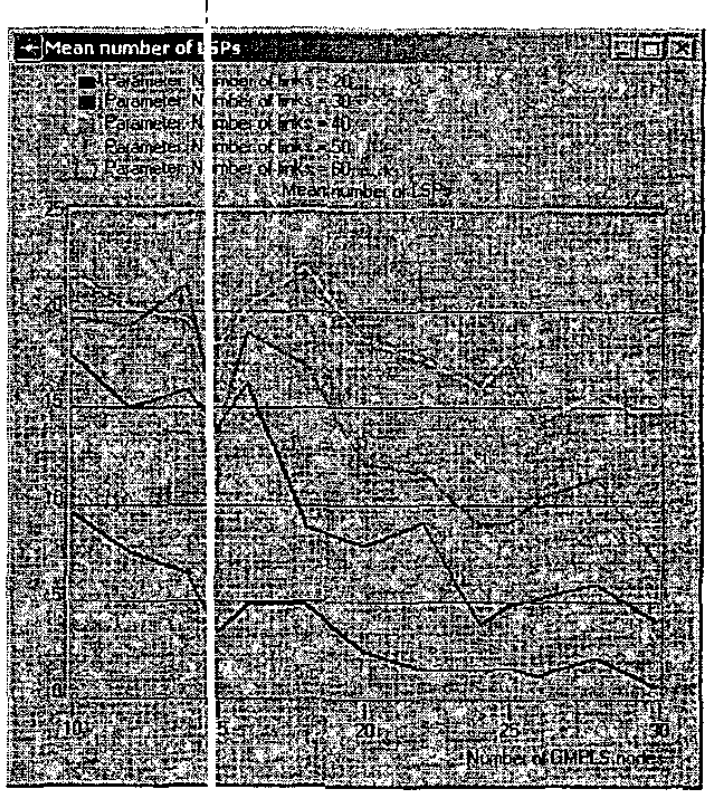

Figure 10: Average, number of LSPs through networks of various sizes. 


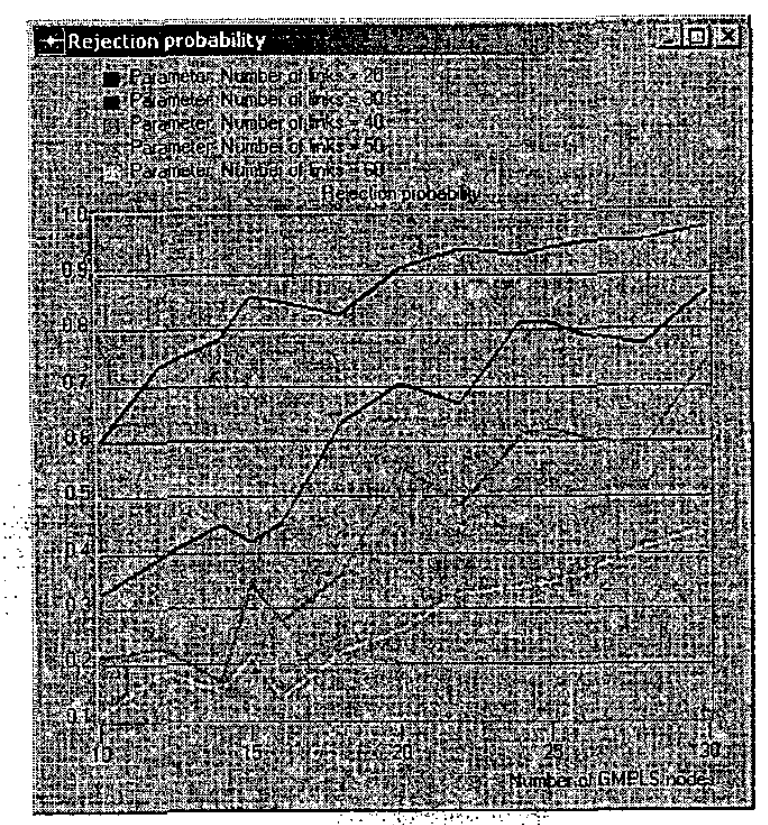

Figure 11: Rejection (blocking) probability for a number of different network sizes. $\therefore \because \cdots$

The GMPLS model has been integrated with the OPNET MPLS models. Figure 12 shows a scenario where GMPLS is used is the core of a MPLS network.

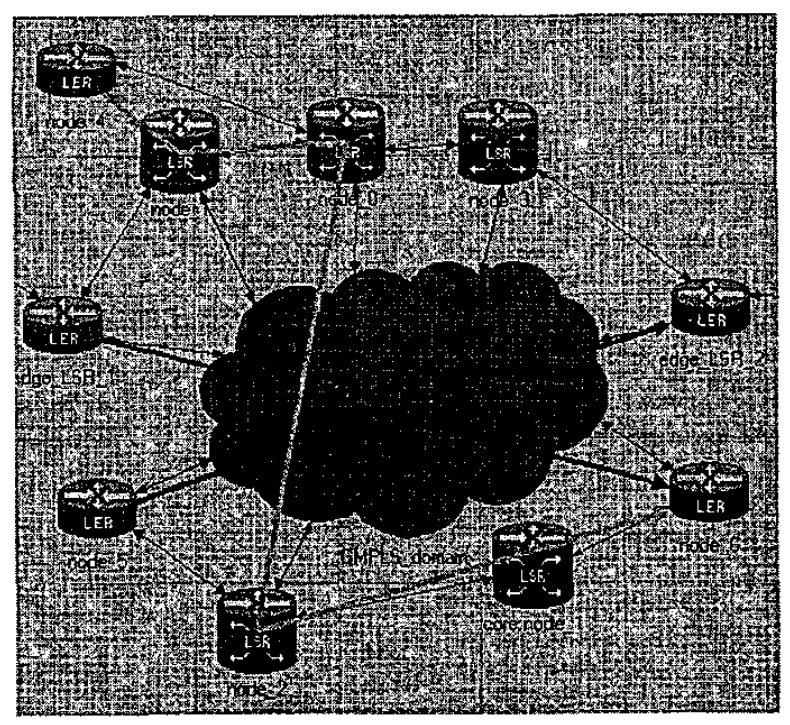

Figure 12: The GMPLS models are fully integrated with the OPNET MPLS models.

MPLS setup request are propagated to all involved nodes by the LDP protocol. The GMPLS model responds to these setup request by setting up a path. GMPLS path setups are reported in the OPNET simulation log. Hence an end-to-end path can cross as well MPLS and GMPLS domains in the network. In a typical scenario, where GMPLS is used in the core, the path will thus be MPLS-GMPLS/key-MPLS-MPLS.

\section{Modeling the Key MPLS scheme}

The scalability of the scheme is evaluated through simulation of randomly connected networks of various sizes.

The result is shown in Figure 13, where the average and the maximum values represent typical and worst-case values, respectively. It is shown that a label length of about 2 bytes is sufficient to support network sizes of up to 10 all-optical network nodes. Larger networks will generally require longer paths, which are infeasible without optical regeneration.

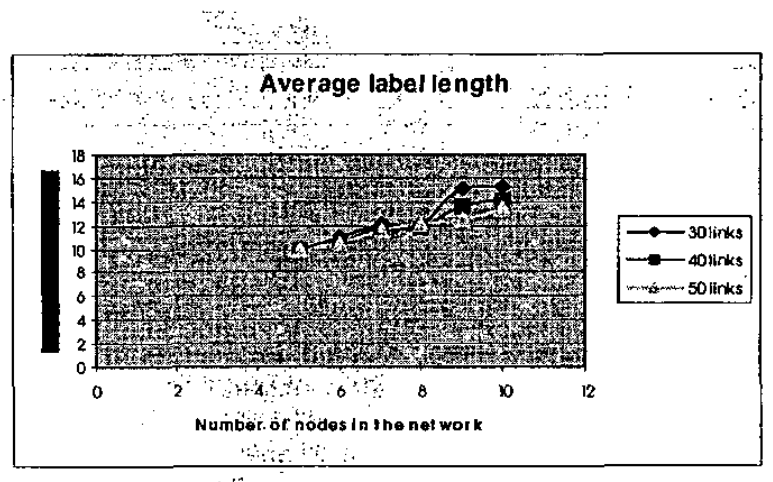

Figure 13: Required size of label field for different network sizes.

Clearly the length increases with networks size, but interestingly enough the length is appropriate for optical networks and does not severely impact the use of network resources.

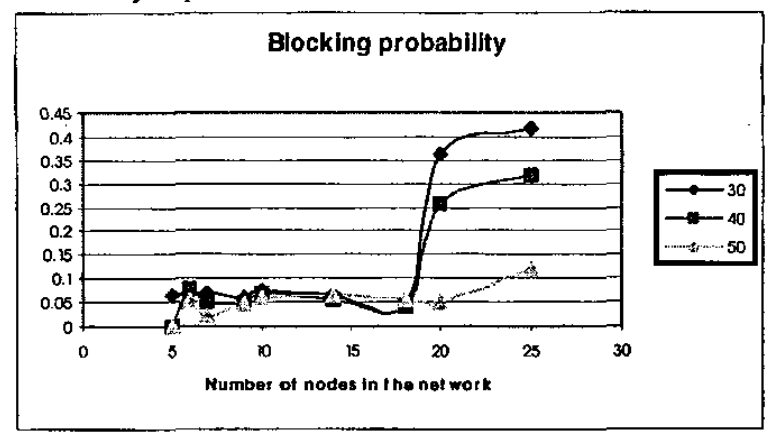

\section{Conclusion}

GMPLS is becoming more and more widely used as a control plane in optical circuit switched networks. Combining GMPLS with MPLS (which in itself can seamlessly integrate a number of packet switched technologies, regardless of protocol) yields an interesting network architecture, which is rather future proof. In this paper a model of such mixed MPLS, GMPLS network has been presented. Path setup through MPLS and GMPLS has been demonstrated and impact of network size on e.g. call rejection probability has been measured. Furthermore simulation on a novel packet forwarding scheme for optical MPLS networks and 
simulation results are presented that shows the feasibility of this scheme.

\section{References}

[Ber2002] L. Berger, Y. Rekhter, "Generalized MPLS Signaling - Implementation Survey", Internet draft, draft-ietf-ccamp-gmpls-signaling-survey-01.txt, May 2002

[Chr2001] H. Christiansen, "Using OPNET To Compure and Analyze Different Traffic- Bundling Schemes", OPNETWork2001.

[Corm990] T. H. Cormen, C. E. Leiserson, R. L. Rivest, "Irtroduction to Algorithms", MIT Press, 1990.

[Ban2001] A. Banerjee, J.Drake, J.P.Lang, B.Turner, K.Kompella, and

Y.Rkhter,"Generalized Multiprotocol Label Switching: An overview of routing and Management Enhancement", IEE communication magazine, January 200I [Veq1996] E.W.Zegura, K.L.Calvest, S.Bhattacherjee "How to model an Internetwork". In proceedings IEEE infocom 1996

[Fen2000] C.Ficnger, E.Limal, U. Gliese, "Statistical Study of the influence of Topology on Wavelength Usage in WDM networks", In proceeding of Optical Fiber Communication Conference (OFC) 2000

[Ros2001a] .E.C., Rosen; et al, "Multiprotocol Label Switching architecture", RFC. 3031, Japuary 2001

[And2001] L. Andersson, et. al., "LDP Specification", RFC 3036, January 2001 · [Rekh2000] Y. Rekhter, "Carrying Label Information in BGP-4", Internet Draft; draft-ietf-mpls-bgp4-mpls-04.txt, work in progress, January 2000

[Jamo1999] B. Jamoussi, et. al., "Constraint-Based LSP Setup using LDP", Internet draft; draft-jetf-mpls-cr-ldp-03.cxt, work in progress, September 1999.

[Brad1997] R. Braden, et al, "Resource ReSerVation Protocol (RSVP) - Version I FunctionaI Specification", RFC 2205. September 1997

[Ros2001b] E.C.Rosen, et al, "MPLS Label Stack Encoding", RFC 3032, January 2001

[Rekh1995] Y. Rekhter, "CIDR and Classful Routing", RFC 1817, August 1995 [Dan]997] S.L Danielsen, Mikkelsen, B., Joergensen, C., Durhuus, T, and Stubkjaer,-KE., "WDM Packet Switch Architectures and Analysis of the Influence of Tuneable Wavelength Converters on the Performance", Joumal of Lightwave Technology, Vol. I5, No. 2, pp. 219-226, 1997.

[Hun2000] Hunter, D.K., Andonovic, I., "Approaches to Optical Internet packet switching", IEEE communications Magazine, September 2000

[Chi1998] Chiaroni, D. Lavigne, B., Jourdan, A., Sotom, M., Hamon, L., et al. "Physical and logical validation of a network based on all-optical packet switching systems". Journal of lightwave technology, vol. 16 \# 12, December 1998 [Wess00l] H. Wessing, T. Fjelde, H. Christiansen, L. Dittmann, "Novel scheme for efficient and cost-effective forwarding of packets in optical networks without header modification", OFC 200!, Techn. Digest, Anaheim, USA, 2001.

[Woll999] Wolfson, D., Hansen, P.B., Kloch, A., Fjelde, T., Janz, C., Coquelin, A., Guillemot, I., Gaboit, F., Poingt, F., Renaud, M., "All-optical regeneration at $40 \mathrm{Gbit} / \mathrm{s}$ in an SOA-based Mach-Zehnder interferometer", in Technical Digest of OFC"99, post deadline paper PD36, San Diego (USA), Feb., 1999

[Wess002] H. Wessing, H. Christiansen, T. Fjelde, L. Dittnıann, "Novel scheme packet forwarding without header modifications in optical networks",Accepted for publication in IEEE JLT, August 2002.

[Chr2002] H.Christiansen, T.Fjelde, H.Wessing, "Novel label processing schemes for MPLS", Optical Networks Magazine. November/December 2002 